\begin{tabular}{|c|c|}
\hline Title & Highly Ordered A nodic A lumina Nanofibers Fabricated via Two Distinct A nodizing Processes \\
\hline Author(s) & Nakajima, Daiki; Kikuchi, Tatsuya; Natsui, Shungo; Suzuki, Ryosuke 0. \\
\hline Citation & $\begin{array}{l}\text { ECS Electrochemistry Letters, 4(5), H14-H17 } \\
\text { https://doi.org/10.1149/2.0021505eel }\end{array}$ \\
\hline Issue Date & 2015 \\
\hline Doc URL & http:/hdl .handle.net/2115/59567 \\
\hline Rights & $\begin{array}{l}\text { () The Electrochemical Society, Inc.2015. All rights reserved. Except as provided under U.S. copyright law, this work } \\
\text { may not be reproduced, resold, distributed, or modified without the express permission of The Electrochemical Society } \\
\text { (ECS). The archival version of this work was published in ECS Electrochemistry Letters. }\end{array}$ \\
\hline Rights(URL) & http://creativecommons.org/icenses/by-nc-nd/4.0/ \\
\hline Type & article \\
\hline File Information & H14.full.pdf \\
\hline
\end{tabular}

Instructions for use 


\title{
Highly Ordered Anodic Alumina Nanofibers Fabricated via Two Distinct Anodizing Processes
}

\author{
Daiki Nakajima, Tatsuya Kikuchi, ${ }^{\mathrm{z}}$ Shungo Natsui, and Ryosuke O. Suzuki \\ Faculty of Engineering, Hokkaido University, Sapporo, Hokkaido, 060-8628, Japan
}

\begin{abstract}
Fabrication of high-density, highly ordered anodic alumina nanofibers was demonstrated via two distinct anodizing processes: porous and fibrous oxide formations. Highly ordered aluminum dimple arrays were fabricated via sulfuric/oxalic acid anodizing and selective porous alumina dissolution. Subsequent pyrophosphoric acid anodizing using the nanostructured aluminum surface caused anodic alumina nanofibers to grow preferentially at the six apexes of the ordered hexagonal aluminum dimples under the appropriate electrochemical conditions. Well-defined, high-density, highly ordered anodic alumina nanofibers with 37-75 nm periodic spacing and at densities of 1.4-5.6 $\times 10^{14} \mathrm{~m}^{-2}$ were successfully fabricated on the aluminum surface via two distinct anodizing processes. (C) The Author(s) 2015. Published by ECS. This is an open access article distributed under the terms of the Creative Commons Attribution Non-Commercial No Derivatives 4.0 License (CC BY-NC-ND, http://creativecommons.org/licenses/by-nc-nd/4.0/), which permits non-commercial reuse, distribution, and reproduction in any medium, provided the original work is not changed in any way and is properly cited. For permission for commercial reuse, please email: oa @electrochem.org. [DOI: 10.1149/2.0021505eel] All rights reserved.
\end{abstract}

Manuscript submitted February 11, 2015; revised manuscript received February 27, 2015. Published March 17, 2015.

Anodizing is a very important surface finishing of aluminum and its alloys for corrosion protection, ${ }^{1-3}$ electronic devices, ${ }^{4,5}$ and micro/nano-fabrication. ${ }^{6-9}$ Anodic aluminum oxide formed by anodizing has typically been classified into two different groups with distinct nanomorphology characteristics: barrier and porous oxides. ${ }^{10,11} \mathrm{An}$ anodic barrier oxide can be obtained by anodizing in neutral solutions, and it possesses a thin, compact amorphous layer. ${ }^{12,13}$ In contrast, an anodic porous oxide can be formed by anodizing in acidic solutions and consists of numerous thick nanoscale hexagonal cells with nanopores at the center. ${ }^{14-16}$ Notably, self-ordering of the hexagonal cells can be achieved at the maximum voltage required to induce a high current density without burning, resulting in the formation of highly ordered porous alumina. ${ }^{17-22}$ Anodic alumina possessing nanopores with a wide range of diameters, from tens to several hundreds of nanometers, is assembled during these anodizing techniques. ${ }^{23-25}$ Porous oxide films with nanopores can also be formed on the aluminum substrate via anodizing in alkaline solutions, but it is difficult to obtain a well-ordered porous oxide film. ${ }^{26-28}$ Because the nanomorphology of an anodic oxide is essentially limited to barrier or porous oxides, the discovery of an additional anodic oxide with different nanofeatures would expand the applicability of anodizing.

Very recently, we reported a third-generation anodic oxide, anodic alumina nanofibers, fabricated via anodizing in concentrated pyrophosphoric acid. ${ }^{29}$ During this anodizing process, alumina nanofibers measuring less than $10 \mathrm{~nm}$ in diameter were formed at the triple points of the honeycomb-shaped anodic oxide on the aluminum substrate. The aluminum surface covered with nanofibers exhibits ultra-fast superhydrophilic properties. However, it is difficult to apply high voltages above $100 \mathrm{~V}$ during pyrophosphoric acid anodizing due to the active anodic dissolution of the aluminum substrate. Therefore, highly ordered alumina nanofibers could not be obtained by pyrophosphoric acid anodizing at the high voltage required to induce self-ordering, as reported previously.

Herein, we demonstrate highly ordered anodic alumina nanofibers fabricated via two distinct advanced anodizing processes based on a porous oxide formation, selective oxide dissolution, and alumina nanofiber formation. In these processes, the growth of the alumina nanofibers was induced from the ordered convex parts located at the apexes of the hexagonal aluminum dimple array. Novel high-density, highly ordered anodic alumina nanofibers were successfully obtained on the aluminum substrate over a large area.

\section{Experimental}

The fabrication processes of the highly ordered anodic alumina nanofibers via two distinct anodizing processes are described in Fig. 1.

${ }^{\text {zE} E-m a i l: ~ k i k u @ e n g . h o k u d a i . a c . j p ~}$
High-purity aluminum foils and plates (99.99 wt\%, 110-400 $\mu \mathrm{m}$ thick, Showa Aluminum and Nippon Light Metal, Japan) were ultrasonically degreased in $\mathrm{C}_{2} \mathrm{H}_{5} \mathrm{OH}$ for $10 \mathrm{~min}$ and then electrochemically polished in a $13.6 \mathrm{M} \mathrm{CH}_{3} \mathrm{COOH} / 2.56 \mathrm{M} \mathrm{HClO}_{4}$ solution $(280 \mathrm{~K})$ at $28 \mathrm{~V}$ for $1 \mathrm{~min}$ (Fig. 1a). Then, the electropolished specimens were anodized in the following electrolyte solutions to form ordered porous alumina (Fig. 1b): a) $0.5 \mathrm{M}$ sulfuric acid $(283 \mathrm{~K})$ at $25 \mathrm{~V}$ for $6 \mathrm{~h}$; and b) $0.3 \mathrm{M}$ oxalic acid $(293 \mathrm{~K})$ at $50 \mathrm{~V}$ for $60 \mathrm{~min}$. The anodized specimens were immersed in a $0.20 \mathrm{M} \mathrm{CrO}_{3} / 0.51 \mathrm{M} \mathrm{H}_{3} \mathrm{PO}_{4}$ solution $(353 \mathrm{~K})$ to selectively dissolve the porous alumina. Therefore, an aluminum dimple array corresponding to the bottom shape of the porous alumina was exposed to the surface (Fig. 1c). ${ }^{30}$ After chemical dissolution, the aluminum specimens were anodized again in concentrated pyrophosphoric acid (74.0-78.0\%, Kanto Chemical, Japan, $293 \mathrm{~K}$ ) at $25-50 \mathrm{~V}$ for $9 \mathrm{~min}$ to form the ordered alumina nanofibers (Fig. 1d). A single-step pyrophosphoric acid anodizing process using electropolished aluminum was also carried out for comparison.

The nanomorphology of the anodized specimens was examined by field emission scanning electron microscopy (FE-SEM, JSM$6500 \mathrm{~F}$ and JIB-4600F/HKD, JEOL, Japan). A thin platinum layer was coated on the anodized specimens by sputtering prior to the SEM observations.

\section{Results and Discussion}

Figure 2 shows SEM images of the anodic alumina nanofibers fabricated via the single-step pyrophosphoric acid anodizing. An electropolished specimen was anodized in concentrated pyrophosphoric acid at $293 \mathrm{~K}$ and $75 \mathrm{~V}$ for $315 \mathrm{~s}$. The aluminum specimen was covered with numerous alumina nanofibers on the thin honeycomb oxide, and the nanomorphology of the anodized surface was clearly different from that of the typical anodizing processes used for barrier and porous oxide formation. However, the regularity of the nanofibers was extremely poor, and disordered nanofibers were distributed on the surface. Pyrophosphoric acid anodizing under any electrochemical condition without active aluminum dissolution at a high voltage resulted in the formation of the disordered alumina nanofibers. Therefore, we achieved the formation of ordered alumina nanofibers using the two distinct anodizing processes, as described in Fig. 1.

Figure 3 a shows the SEM images of the aluminum substrate after oxalic acid anodizing at $50 \mathrm{~V}$ for $60 \mathrm{~min}$ and the subsequent selective oxide dissolution. A highly ordered aluminum dimple array measuring approximately $130 \mathrm{~nm}$ in diameter was distributed on the aluminum substrate via self-ordered anodizing (Fig. 3a, left). Note that the sides of the hexagonal dimples were not flat when the viewing angle was altered (tilted), and a convex feature formed at the apexes of each hexagonal dimple array, as indicated by the yellow arrow (Fig. 3a, right). These convex features correspond to the last anodizing point 
Ordered porous alumina

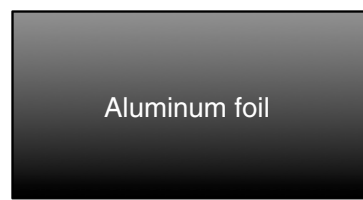

a) Electropolishing

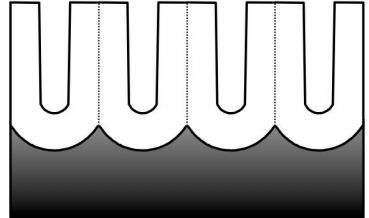

b) Porous alumina formation

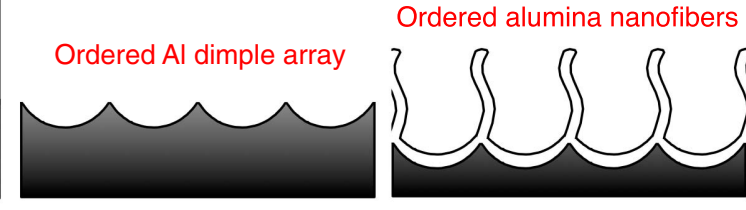

c) Oxide dissolution d) Alumina nanofiber formation

Figure 1. The strategy for the fabrication of highly ordered anodic alumina nanofibers: a) Electropolishing of the aluminum surface; b) the anodic porous alumina formation via the first anodizing; c) the dimple array formation via the selective dissolution of the anodic oxide; and d) the anodic alumina nanofiber formation via the second anodizing.

surrounded by the hexagonal alumina cells (Fig. 1b). A similar highly ordered aluminum dimple array $64 \mathrm{~nm}$ in diameter was also fabricated via sulfuric acid anodizing at $25 \mathrm{~V}$. For the fabrication of highly ordered anodic alumina nanofibers, the growth of the anodic oxide was induced from these convex aluminum features during pyrophosphoric acid anodizing.

The change in the current density over time during pyrophosphoric acid anodizing using the nanostructured aluminum specimen with the ordered dimple array is shown in Figure $3 \mathrm{~b}$. During the second pyrophosphoric acid anodizing step, the applied voltage was adjusted to $\mathrm{U}=50 \mathrm{~V}$, which is the same voltage used during the first oxalic acid anodizing step. The current density increased and decreased rapidly in the initial period of $10 \mathrm{~s}$, and then gradually decreased with anodizing time. Figure $3 \mathrm{c}$ shows an SEM image of the alumina nanofibers fabricated via pyrophosphoric acid anodizing using the nanostructured aluminum specimen. The anodic alumina nanofibers were formed selectively at the apexes of each hexagonal dimple array, and well-ordered alumina nanofibers with $75 \mathrm{~nm}$ periodic spacing and approximately $100 \mathrm{~nm}$ in length were successfully fabricated via two distinct anodizing processes, as described by the schematic in Fig. 1. The density of the nanofibers was measured to be $1.4 \times 10^{14} \mathrm{~m}^{-2}$, which is extremely high. The diameter of each alumina nanofiber was also measured as approximately $20 \mathrm{~nm}$ via the SEM images. However,
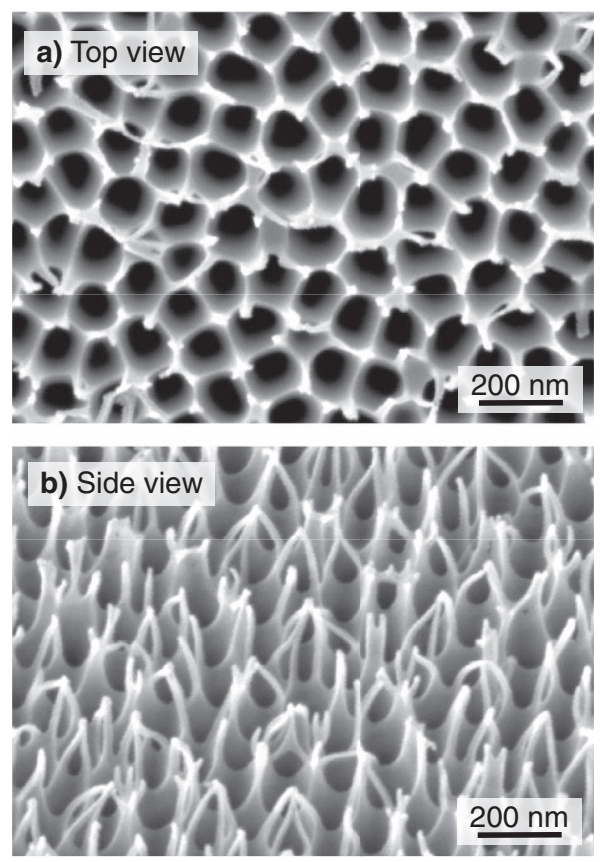

Figure 2. SEM images of the surface of a specimen anodized in pyrophosphoric acid at $75 \mathrm{~V}$ for $315 \mathrm{~s}$. Numerous disordered anodic alumina nanofibers were formed on the aluminum specimen.

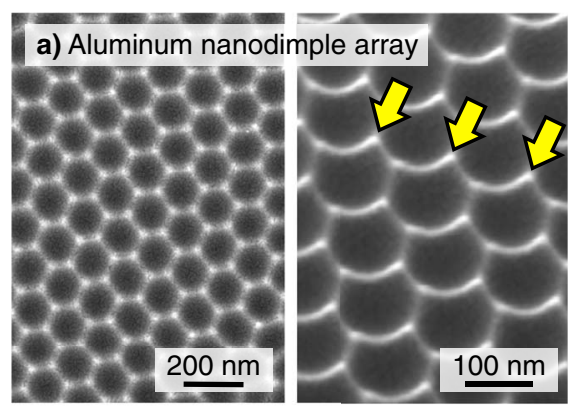

b) Current-time transient
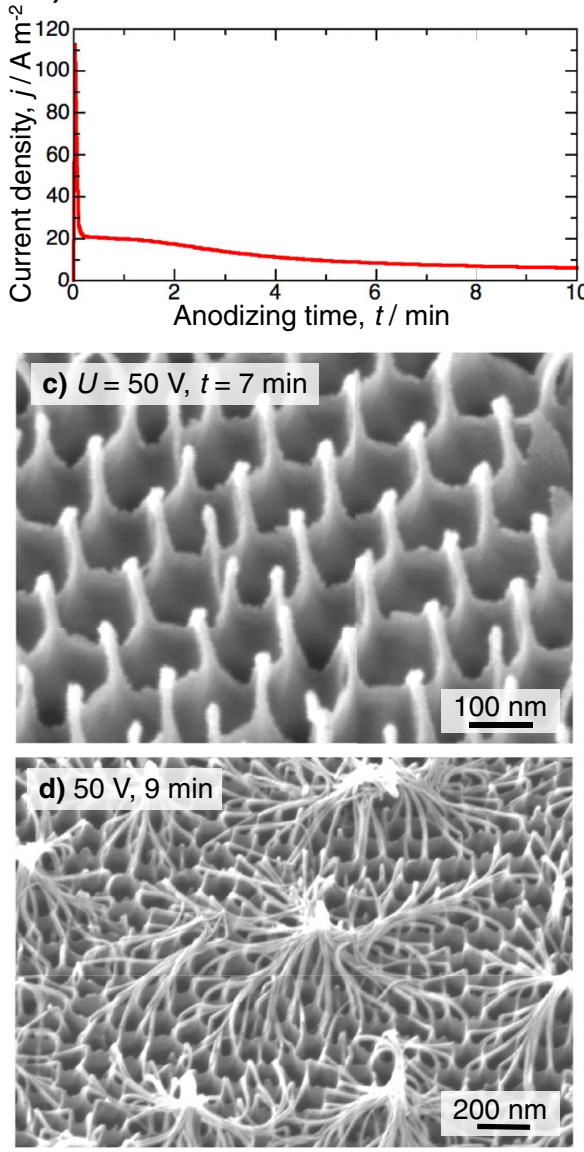

Figure 3. a) A highly ordered aluminum dimple array fabricated using oxalic acid anodizing and subsequent selective oxide dissolution. b) Change in current density over time via pyrophosphoric acid anodizing at constant voltage of $50 \mathrm{~V}$. c) Highly ordered anodic alumina nanofibers fabricated on the ordered dimple array via pyrophosphoric acid anodizing at $50 \mathrm{~V}$ for $7 \mathrm{~min}$. d) Bundled nanostructures with longer nanofibers fabricated via further pyrophosphoric acid anodizing for $9 \mathrm{~min}$. 

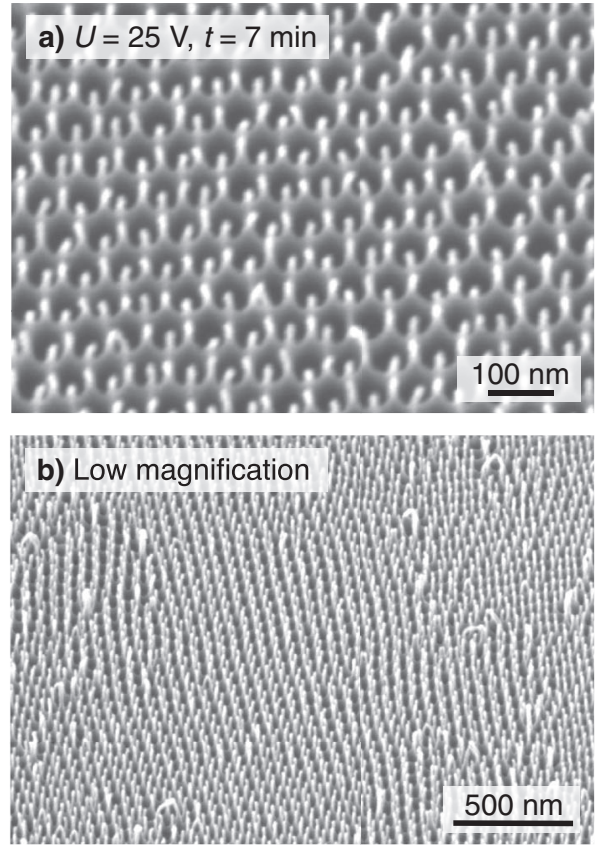

Figure 4. a) High- and b) low-magnification of the highly ordered anodic alumina nanofibers fabricated via two distinct anodizing processes in sulfuric and pyrophosphoric acid at $25 \mathrm{~V}$.

the surface of the anodized specimen was covered by a thin platinum electroconductive layer before the SEM characterization, and it is likely that the actual diameter is smaller than that observed from Fig. 3c. In the previous study, the diameter was measured as less than $10 \mathrm{~nm}$ using high-resolution transmission electron microscopy. ${ }^{29}$ During the typical two-step anodizing process for the formation of ordered porous alumina, the nanopores are preferentially generated at the bottom of the dimple array and grow downward from the surface (Fig. 1c). In our case, for the ordered alumina nanofiber formation, it is noted that the nanofibers were preferentially generated at the top apexes of the dimple array and grew upward from the surface. Therefore, the formation behaviors of the anodic alumina nanofibers and the porous alumina possess a mirror image relationship.

As the anodizing time was increased to 9 min (Fig. 3d), the nanofibers increased in length to several hundred nanometers. This rapid growth rate of the alumina nanofibers can be explained by the growth behavior in the initial stage of pyrophosphoric acid anodizing. ${ }^{29}$ Anodic alumina nanofibers grow through the formation of barrier and honeycomb oxides and the subsequent selective dissolution of honeycomb oxide. As a result, various nanofibers were tangled and bundled together due to their flexibility, and the interesting nanofeatures that formed on the aluminum substrate were covered by alumina nanofibers having the capability to move. The surface nanofeatures were very similar to those obtained from the single-step pyrophosphoric acid anodizing, ${ }^{29}$ and long-term anodizing is inadequate for the fabrication of highly ordered nanofiber structures. It is speculated that the alumina nanofibers float freely in the pyrophosphoric acid solution during anodizing.

Highly ordered anodic alumina nanofibers with even greater density were also fabricated via sulfuric acid anodizing and subsequent pyrophosphoric acid anodizing. Sulfuric acid anodizing under the selfordering condition of $U=25 \mathrm{~V}$ provided a highly ordered aluminum dimple array measuring $64 \mathrm{~nm}$ in diameter, which is smaller than that obtained via oxalic acid anodizing. Figures $4 \mathrm{a}$ and $4 \mathrm{~b}$ show the highand low-magnification SEM images of the nanostructured aluminum surface fabricated via the two-step sulfuric and pyrophosphoric acid anodizing process. Well-defined, high-density, highly ordered anodic alumina nanofibers with $37 \mathrm{~nm}$ in period were clearly distributed on the aluminum surface and covered a wide region of the surface (although, several irregular imperfections were observed due to the simple two-step anodizing). Fabrication of an ordered imperfectionfree alumina nanofiber array will be achieved by first anodizing using nanoimprinting on the aluminum specimen. The density of the alumina nanofibers was greater than that obtained in Fig. 3 and was measured as $5.6 \times 10^{14} \mathrm{~m}^{-2}$. Thus, our two distinct anodizing processes can be easily realized for the fabrication of high-density, highly ordered anodic alumina nanofibers $\left(1.4-5.6 \times 10^{14} \mathrm{~m}^{-2}\right.$, periodic spacing: $37-75 \mathrm{~nm}$ ) on the aluminum surface.

Recently, formation of alumina nanofibers or nanowires based on anodizing and subsequent chemical etching has been reported by several research groups. ${ }^{31-33}$ However, it is difficult to accurately control the regularity, length, and density of ultra-narrow alumina nanofibers. Precise nanofiber-structuring was achieved via our two distinct anodizing processes including pyrophosphoric acid anodizing on the aluminum dimple array surface.

In summary, ultra high-density, highly ordered anodic alumina nanofiber arrays were successfully fabricated via two distinct anodizing processes including anodic porous alumina formation, selective oxide dissolution, and subsequent anodic alumina nanofiber formation. The density and length of the alumina nanofibers were accurately controlled by the choice of electrolyte in the first anodizing process and by the processing time in the second anodizing process. Our novel ordered alumina nanofibers have various nanoapplications including wettability control and catalyst supports. The aluminum surface covered by alumina nanofibers exhibited ultra-fast superhydrophilic behavior with less than $1^{\circ}$ within 1 second, as previously reported. ${ }^{29}$ Therefore, the superhydrophilic property for nanostructured wettability control will be related to regularity, density, and length of the ordered alumina nanofibers via further investigations.

\section{Conclusions}

This letter describes highly ordered anodic alumina nanofibers fabricated via two distinct anodizing processes in the typical sulfuric/oxalic acid and subsequent pyrophosphoric acid solutions. The anodic alumina nanofibers grew in a disordered fashion on the electropolished aluminum substrate via a single-step pyrophosphoric acid anodizing process. In contrast, the ordered growth of alumina nanofibers was induced from the ordered convex aluminum parts located at the six apexes of the ordered hexagonal dimple array during the distinct two-step anodizing process. Therefore, high-density, highly ordered anodic alumina nanofibers can be successfully fabricated via dimple array formation and subsequent pyrophosphoric acid anodizing processes.

\section{Acknowledgments}

This study was conducted at Hokkaido University and was supported by the "Nanotechnology Platform" Program of the Ministry of Education, Culture, Sports, Science, and Technology (MEXT), Japan. The work was financially supported by the Japan Society for the Promotion of Science (JSPS) "KAKENHI" and Toyota Physical \& Chemical Research Institute Scholars.

\section{References}

1. F. Mansfeld and M. W. Kendig, J. Electrochem. Soc., 135, 828 (1988)

2. S. Moon, Y. Nam, C. Yang, and Y. Jeong, Corr. Sci., 53, 1547 (2011).

3. S. Moon and Y. Jeong, Corr. Sci., 51, 1506 (2009).

4. H. Uchi, T. Kanno, and R. S. Alwitt, J. Electrochem. Soc., 148, B17 (2001).

5. T. Hasebe and R. S. Alwitt, J. Electrochem. Soc., 154, C626 (2007).

6. W. Lee, R. Ji, U. Gösele, and K. Nielsch, Nat. Mater, 5, 741 (2006).

7. J. Martín, M. Martín-González, J. F. Fernández, and O. Caballero-Calero, Nat. Commun, 5, 5130 (2014).

8. K.-T. Tsai, C.-Y. Liu, H.-H. Wang, T.-Y. Liu, M.-Y. Lai, J.-H. He, and Y.-L. Wang, Nanotechnology, 25, 335301 (2014).

9. Z. Kato, M. Sakairi, and H. Takahashi, J. Electrochem. Soc., 148, C790 (2001).

10. W. Lee and S.-J. Park, Chem. Rev., 114, 7487 (2014).

11. G. D. Sulka, in Nanostructured Materials in Electrochemistry, A. Eftekhari, Editor, p. 1, Wiley-VCH VerlagGmbH \& Co. KGaA, Germany (2008)

12. K. Kobayashi and K. Shimizu, J. Electrochem. Soc., 135, 908 (1988)

13. K. Shimizu, G. E. Thompson, and G. C. Wood, Electrochim. Acta, 27, 245 (1982). 
14. X. Qin, J. Zhang, X. Meng, C. Deng, L. Zhang, G. Ding, H. Zeng, and X. Xu, Appl. Surf. Sci., 328, 459 (2015).

15. T. Kikuchi, O. Nishinaga, S. Natsui, and R. O. Suzuki, ECS Electrochem. Lett, 3 , C25 (2014).

16. M. Michalska-Domańska, M. Norek, W. J. Stępniowski, and B. Budner, Electrochim. Acta, 105, 424 (2013)

17. H. Masuda and F. Hasegawa, J. Electrochem. Soc., 144, L127 (1997).

18. H. Asoh, K. Nishio, M. Nakao, T. Tamamura, and H. Masuda, J. Electrochem. Soc., 148, B152 (2001).

19. S. Ono, M. Saito, and H. Asoh, Electrochim. Acta, 51, 827 (2005)

20. O. Nishinaga, T. Kikuchi, S. Natsui, and R. O. Suzuki, Sci. Rep., 3, 2748 (2013).

21. T. Kikuchi, O. Nishinaga, S. Natsui, and R. O. Suzuki, Electrochim. Acta, 137, 728 (2014).

22. T. Kikuchi, O. Nishinaga, S. Natsui, and R. O. Suzuki, Electrochim. Acta, 156, 235 (2015).

23. J. Martin, C. V. Manzano, O. Caballero-Calero, and M. Martin-Gonzalez, ACS Appl. Mater. Interfaces, 572 (2013)
24. C. V. Manzano, J. Martin, and M. S. Martin-Gonzalez, Micropor. Mesopor. Mat., 184 177 (2014)

25. A. P. Li, F. Müller, A. Birner, K. Nielsch, and U. Gösele, J. Appl. Phys, 846023 (1998).

26. A. O. Araoyinbo, M. N. Ahmad Fauzi, S. Sreekantan, and A. Aziz, Asian J. Mater. Sci., 2, 63 (2010).

27. L. O. Snizhko, A. L. Yerokhin, N. L. Gurevina, D. O. Misnyankin, A. Pilkington, A. Leyland, and A. Matthews, Electrochim. Acta, 50, 5458 (2005).

28. H. Noguchi and A. Sano, J. Surf. Fin. Soc. Jpn., 49, 649 (1998).

29. T. Kikuchi, O. Nishinaga, D. Nakajima, J. Kawashima, S. Natsui, N. Sakaguchi, and R. O. Suzuki, Sci. Rep., 4, 7411 (2014).

30. T. Kikuchi, T. Yamamoto, and R. O. Suzuki, Appl. Surf. Sci., 284, 907 (2013).

31. B. E. Alpysbayeva, Kh. A. Abdullin, J. K. Kalkozova, R. R. Nemkayeva, and N. R. Guseinov, Inorg. Mater, 51, 129 (2015).

32. A. Nazemi, A. Najafian, and S. A. S. Sadjadi, Superlattice. Microst., 81, 1 (2015).

33. S. Peng, D. Tian, X. Miao, X. Yang, and W. Deng, J. Colloid Interf. Sci., 409, 18 (2013). 